\title{
Frequency response analysis of hydroelectric power plants with influence from a non-linearized frictional damping and the turbine characteristics $\dagger$
}

\section{H. BREKKE:}

Keywords: frequency response, hydroelectric power plant control, water turbines

The main purpose of the dissertation has been to establish a complete stability analysis of a hydroelectric power plant. The most important part in this study has been to establish a theory for the damping of oscillatory flow in tunnels and pipes. The influence of the turbine characteristics is also important and has been included by differentiation of the turbine equation. The partial derivative values can be found by means of the characteristic diagram of the turbine. Special attention is paid to establishing an empirical friction function for tunnels with rough walls. The author has based his theory on experimental tests carried out for damping of sea waves on rough beds, and the friction factor is a function of both frequency, amplitudes, cross section area and roughness of the wall (Jonsson 1978). Further, the damping of oscillations in shafts leading to tunnels taking into account the mean velocity in the tunnel has been established.

The analysis is based upon the matrix solution of the Laplace transformed complex equations. The turbine matrix is a $7 \times 7$ matrix which may be connected to matrices for the water level control and the electric grid system.

The analysis is verified by measurements in full scale hydroelectric power plants. It should be noted that open cracks seem to have a great influence on damping of oscillations while cracks which are closed by the rock pressure seem to have no influence on the damping.

Other experimental tests not presented in this article including hydroelectric power plants with Kaplan Bulb turbines, Francis and Pelton turbines show a good agreement with the applied theory for frequencies up to about $3 \mathrm{rad} / \mathrm{sec}$.

\section{Introduction}

The purpose of this condensed version of the author's work (Brekke 1984) has been to highlight the influence of the frictional damping of oscillatory flow in the blasted tunnels which normally form the main conduits in high head hydroelectric power plants. The frictional damping factor has been established as a function of flow amplitudes and frequency. In addition the influence of the turbine characteristics plays an important role. The author has verified that a linearization of the steady state characteristic diagrams may be used for frequencies up to about 6.28 $\mathrm{rad} / \mathrm{sec}(=1 \mathrm{~Hz})$ for mean size turbines.

The influence on the damping of oscillations from open cracks in the tunnel system is also illustrated in addition to the damping in small shafts connected to conduits with a steady state velocity. Because the frictional damping is found to be a non-linear function of the flow amplitudes the problem is solved by matrix solution of matrices of Laplace transformed equations.

Received 15 October 1984.

$\dagger$ This paper is a brief summary of a dissertation (Brekke 1984).

$\ddagger$ Kvaerner Brug a.s., P.O. Box 3610 G.b., 0135 Oslo 1, Norway. 


\section{The Laplace transformed equation for tunnels or pipes}

\subsection{The general equation}

The equations for a cylindrical conduit with elastic walls and fluid yields when Laplace transformation is introduced (Brekke 1984, Brekke 1974) are:

$$
\partial h / \partial l=Q_{0} /\left(H_{0} g A\right) \cdot(s+k) q
$$

and

$$
\partial q / \partial l=A H_{0} g /\left(a^{2} Q_{0}\right) s h
$$

where

$$
k=\pi D \tau /\left(\rho Q_{0}|q|\right) \quad(\tau=\text { shear force })
$$

and

$$
a=((\partial A / \partial p)(\rho / A)+\partial \rho / \partial p)^{-0 \cdot 5}
$$

\subsection{The frictional damping factor $\mathrm{k}$}

The author's main study had been concentrated on eqn. (3) where the shear force for rough tunnel walls has been of special interest. For oscillatory flow superimposed on a steady state flow the shear force $\tau$ may consist of one term as a function of the steady state flow and another term as a function of the flow oscillations.

\subsection{The steady state term}

The influence from the steady state flow has been based on linearizing the steady state shear force.

For tunnels, the steady state term $k$ based on Mannings number yields:

$$
k=2 g Q_{t} /\left(M^{2} R_{h}^{4 / 3} A\right)
$$

where $Q_{t}=$ the steady state flow, $R_{h}=$ hydroelectric radius and $A=$ cross section.

$$
k_{t}=4 \lambda Q_{t} /\left(\pi D^{3}\right)
$$

where $\lambda=$ Moody friction constant and $D=$ pipe diameter.

\subsection{The term for pure oscillatory flow in smooth pipes}

The oscillatory flow for smooth pipes is based on the work by Köngeter (1980)

$$
\tau=0 \cdot 85(\alpha / D)^{-0 \cdot 33} \rho(\alpha \omega)^{2}(D \sqrt{ }(\omega / v)) /(\alpha D \omega / v)
$$

or when substituting for $\alpha=Q_{0}|q| /(A \omega / v)$

$$
\tau=0.85 \rho(D \omega)^{0 \cdot 33} \sqrt{ }(\omega v)\left(Q_{0}|q| / A\right)^{0.67}
$$

Partly in order to obtain similarity with the frictional damping of rough tunnels (see Fig. 1), and partly based upon the plotted measured results in Köngeter's work (1980), the shear force for $Q_{0}|q| /(\omega A D) \leqslant 0 \cdot 1447$ is assumed to be:

$$
\tau=\left(2 \cdot 665-7 \cdot 3 Q_{0}|q| /(\omega A D)\right) \pi D \sqrt{ }(\omega v) / A
$$

Over the actual range of frequencies and amplitudes the phase shift will be const. $=(\pi / 4)$ between the flow amplitudes and the shear force: 


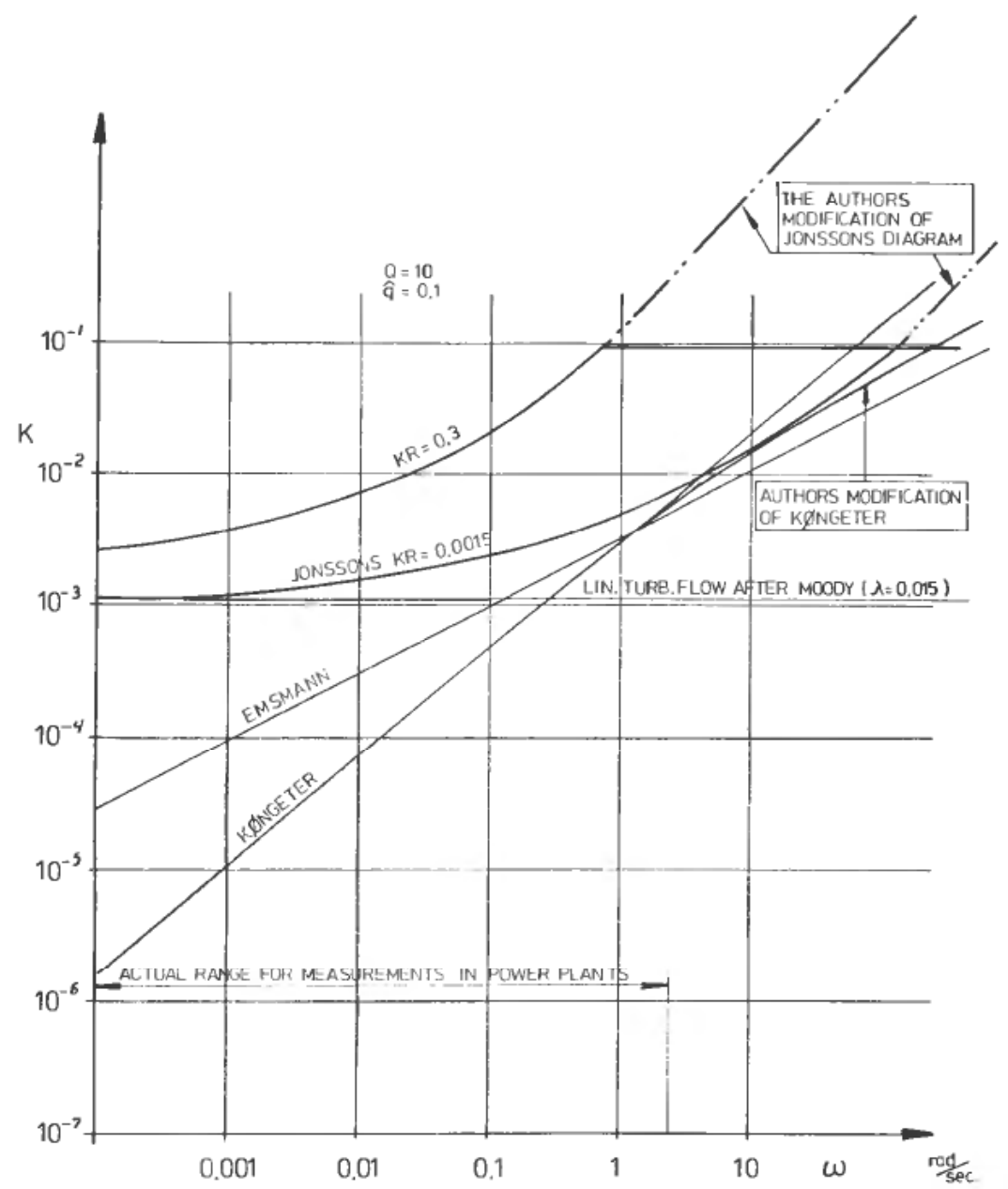

Figure 1. The friction function $K$ as a function of $\omega$ with $A=3.14 \mathrm{~m}^{2}(D=1.0 \mathrm{~m}), Q_{0}=10$ $\left(\mathrm{m}^{3} / \mathrm{sec}\right), \hat{q}=0 \cdot 1$ and $v=10^{-6}\left(\mathrm{~m}^{3} / \mathrm{sec}\right)$.

\subsection{The term for pure oscillatory flow in rough tunnels}

The study of the shear force of oscillatory flow on rough walls has in the author's work (Brekke 1984) been based on a work by Jonsson (1978). The purpose of Jonsson's work was the damping of sea waves on a rough sea bed.

The shear force equation in this work yielded:

$$
\tau=f \frac{1}{2} \rho C_{m}^{2}
$$

where $C_{m}=$ oscillatory velocity amplitude and $f=$ friction factor.

In the author's work a formula for $f$, presented by Swart in Jonsson (1978), was used for $\left(C_{m} / k_{r} \omega\right) \leqslant 1 \cdot 57$.

$$
f=\exp \left((-5 \cdot 977+5 \cdot 213)\left(C_{m} /\left(k_{r} \omega\right)\right)-0 \cdot 194\right)
$$

Here $k_{r}=$ a roughness factor which was determined by means of the head loss in the tunnels in Brekke (1984). Here $C_{m}=Q_{0}|q| / A$. 
For $C_{m} / k_{r} \omega>1.57$ the author established the following formula based on measurements in the Norwegian Power Plant Tafjord, (Brekke 1984).

$$
f=0 \cdot 4725\left(C_{m} /\left(k_{r} \omega\right)\right)^{-1 \cdot 0}
$$

The phase shift may, according to Brekke (1984), be set to be constant $=(\pi / 8)$ for the actual range of flow amplitudes and frequencies used in the study of hydroelectric power plants.

In the author's work (Brekke 1984), $C_{m}=Q_{0}|q| / A$ and $k r$ was determined by the following empirical equation:

$$
k_{r}=(400 / A)\left(0 \cdot 192 \cdot \ln \left(29 \cdot 9 /\left(M^{2} A^{0 \cdot 1667}\right)\right)+1 \cdot 146\right)^{5 \cdot 155}
$$

where $M=$ Manning's number and $A=$ cross section area of tunnel.

The friction factor $k$ for pure oscillatory flow, based on different theories for smooth pipes and rough tunnels, is illustrated versus the oscillatory frequency in Fig. 1 and versus the flow amplitude $|q|=\Delta Q / Q_{0}$ in Fig. 2, where $Q_{0}=10 \mathrm{~m}^{3} / \mathrm{sec}$ for a cross section $A=3 \cdot 14 \mathrm{~m}^{3} / \mathrm{sec}$.

In both Fig. 1 and Fig. 2 the author's modification of Köngeter's formula and the theory of Jonsson is shown in order to obtain a better similarity for high values

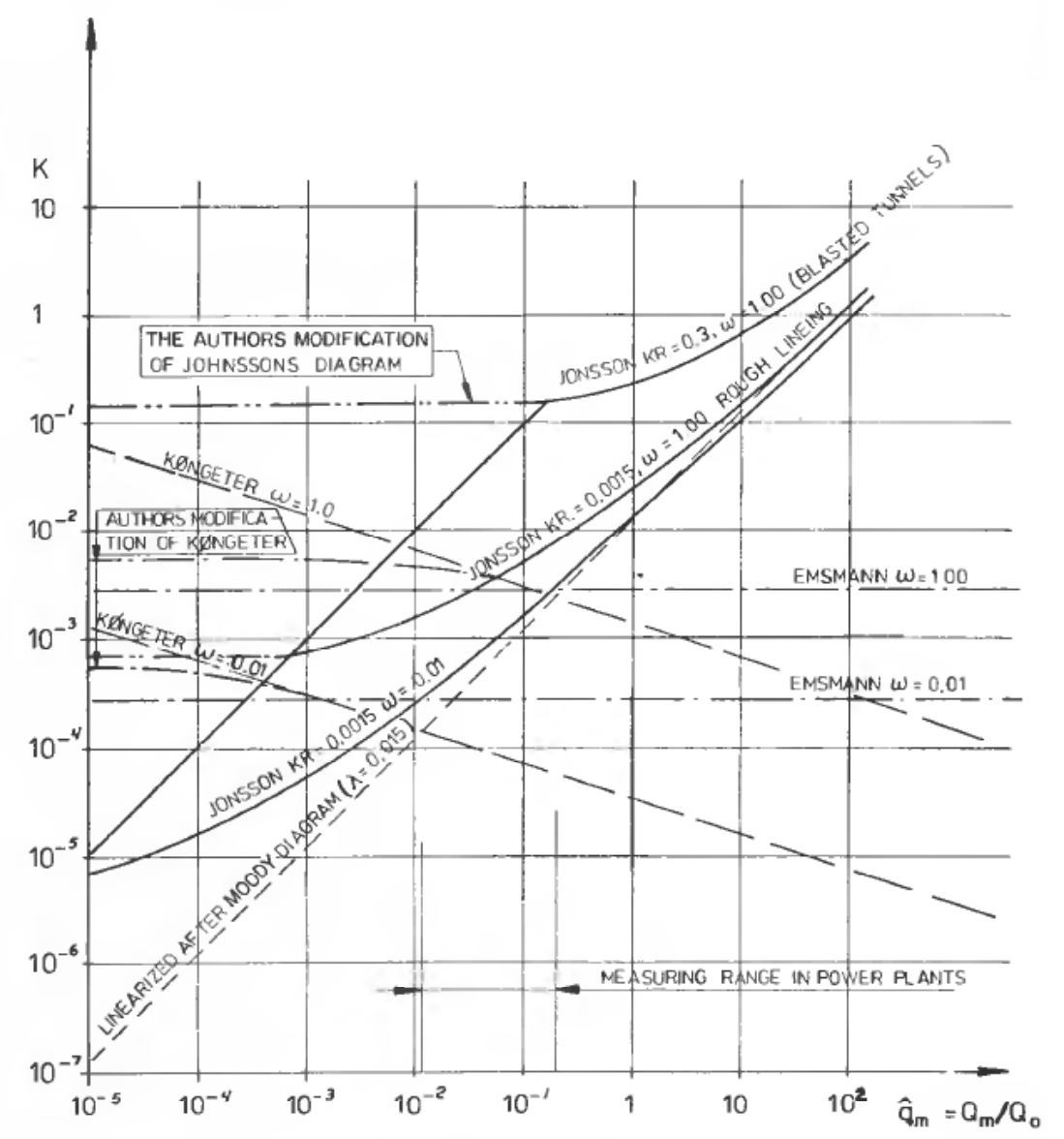

Figure 2. Friction function $K$ as a function of $\hat{q}_{m}$ for cross-section $A=3.14 \mathrm{~m}^{2}$ $(D=1.00 \mathrm{~m}), Q_{0}=10 \mathrm{~m}^{3} / \mathrm{s}$. 
of $\omega$ and small amplitudes. In both figures, friction factors presented by Emsmann referred to in Köngeter (1980) are shown, together with a linearizing of the steady state friction for amplitudes around zero (Brekke 1984).

2.6. The complete formula for the friction factor for oscillatory flow superimposed on the steady state flow

The complete friction factor for smooth pipes will be:

$$
k=\frac{4 \lambda Q_{t}}{\pi D^{3}}+\frac{\pi D \tau}{\rho Q_{0}\left|q_{n-1}\right|} \cos (\pi / 4)+\frac{\pi D \tau}{\rho Q_{0}\left|q_{n-1}\right|} \sin (\pi / 4)
$$

Here $\tau$ is determined by means of eqn. (7) or eqn. (8).

The complete friction factor for rough tunnels will be:

$$
k=\frac{2 g Q_{t}}{M^{2} R_{h}^{4 / 3} A}+\frac{8 f}{\pi D^{3}} Q_{0}\left|q_{n-1}\right| \cos (\pi / 8)+i \frac{8 f}{\pi D^{3}} Q_{0}\left|q_{n-1}\right| \sin (\pi / 8)
$$

where $f$ is determined by means of eqn. (10) or eqn. (11).

\subsection{The matrix for pipe or tunnel elements}

In order to solve eqn. (1) and eqn. (2) when $k=f(q, \omega)$ it has been necessary to establish a matrix solution. The sub matrix for a pipe element when defining the flow out of both ends to be positive and rising pressure to be positive yields:

$$
\left[\begin{array}{cc}
\frac{-s}{2 h_{w} z \tanh (L z / a)} & \frac{s}{2 h_{w} z \sinh (L z / a)} \\
\frac{s}{2 h_{w} z \sinh (L z / a)} & \frac{-s}{2 h_{w} z \tanh (L z / a)}
\end{array}\right] \cdot\left[\begin{array}{l}
h_{L} \\
h_{R}
\end{array}\right]=\left[\begin{array}{l}
q_{L} \\
q_{R}
\end{array}\right]
$$

Here $z=\left(s^{2}+k s\right)^{0.5}, h_{w}=a Q_{0} /\left(2 g A H_{0}\right)$ and $L=$ pipe length.

\section{Matrix for surge shaft or air accumulators}

The matrix for surge shafts will be

$$
\left[\begin{array}{ll}
1 & 0 \\
0 & -i H_{0} A_{\text {eqv }} / Q_{0}
\end{array}\right]\left[\begin{array}{l}
h_{L} \\
h_{R}
\end{array}\right]=\left[\begin{array}{l}
q_{L} \\
q_{R}
\end{array}\right]
$$

where $A_{\text {eqv }}=$ equivalent cross section for an air accumulator.

$$
A_{\text {eqv }}=1 /\left(A_{w}+\kappa H_{a 0} / V_{0}\right)
$$

Here $A_{w}=$ area of water level, $\kappa=$ polytropic constant and $H_{a 0}=$ steady state pressure in the air. (Note: $h_{L}=0$ ).

\section{Matrix for loss in junction of shafts and variations in cross section}

The matrix yields:

$$
\left[\begin{array}{rr}
-1 / k_{\mathrm{res}} & 1 / k_{\mathrm{res}} \\
1 / k_{\mathrm{res}} & -1 / k_{\mathrm{res}}
\end{array}\right] \cdot\left[\begin{array}{l}
h_{L} \\
h_{R}
\end{array}\right]=\left[\begin{array}{l}
q_{L} \\
q_{R}
\end{array}\right]
$$




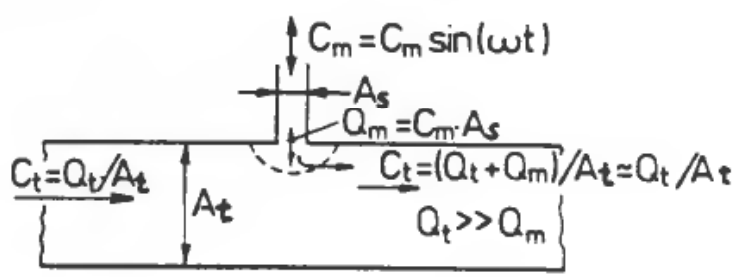

Figure 3. Connection of shaft with cross section $A_{s}$ and a tunnel with cross section $A_{t}$ with steady state velocity $C_{t}$.

where

$$
k_{\text {res }}=\left(k Q_{0} /\left(3 g A_{s} H_{0}\right)\right)|q|+Q_{t} H_{0} /\left(g A_{t} H_{0}\right)
$$

Here $k=1.0$ for sharp corners and $k<1.0$ for rounded corners. The damping influence of the corner of the outlet pipe has been throughly analysed by Knott and Flower (1980). The other values are shown in Fig. 3.

\section{Matrix for local loss caused by variation in cross section area}

The matrix for damping caused by variation in cross section in a tunnel or pipe with steady state flow $=Q_{t}$ yields:

$$
\left[\begin{array}{rr}
-1 / k_{\mathrm{re}} & 1 / k_{\mathrm{re}} \\
1 / k_{\mathrm{re}} & -1 / k_{\mathrm{re}}
\end{array}\right] \cdot\left[\begin{array}{l}
h_{L} \\
h_{R}
\end{array}\right]=\left[\begin{array}{l}
q_{L} \\
q_{R}
\end{array}\right]
$$

where

$$
k_{\mathrm{re}}=\left(2 Q_{0} /\left(H_{0} Q_{t}\right)\right) \zeta\left(1 / A_{1}^{2}-1 / A_{2}^{2}\right) Q_{t}^{2} /(2 g)
$$

$h_{\mathrm{re}}=$ the head loss across the variation in cross section with areas $A_{1}$ and $A_{2}$. $\zeta=$ loss constant, $Q_{\mathrm{t}}=$ steady state loss in the pipe and $Q_{0}$ and $H_{0}$ are steady state conditions at the turbine in the system.

\section{The influence of the turbine characteristics}

The influence of the turbine characteristics may be found by the linearized turbine equation

$$
p=q+h+\eta
$$

where

$$
p=d P / P_{0} \quad q=d Q / Q_{0} \quad h=d H / H_{0} \quad \text { and } \quad \eta=d \eta / \eta_{0}
$$

The values $q$ and $\eta$ may be determined by the following equations:

$$
q=Q_{y} y+Q_{n} n+Q_{\phi} \phi-\frac{1}{2}\left(1-Q_{n}\right) h
$$

where $Q_{y}, Q_{n}$ and $Q_{\phi}$ may be determined from the turbine characteristic diagram as shown in Fig. 4 for a Francis turbine and in Fig. 5(a) and 5(b) for a Kaplan turbine. (Note: $y=d y / y_{0}$ and $\phi=d \phi / \phi_{0}$ ). 


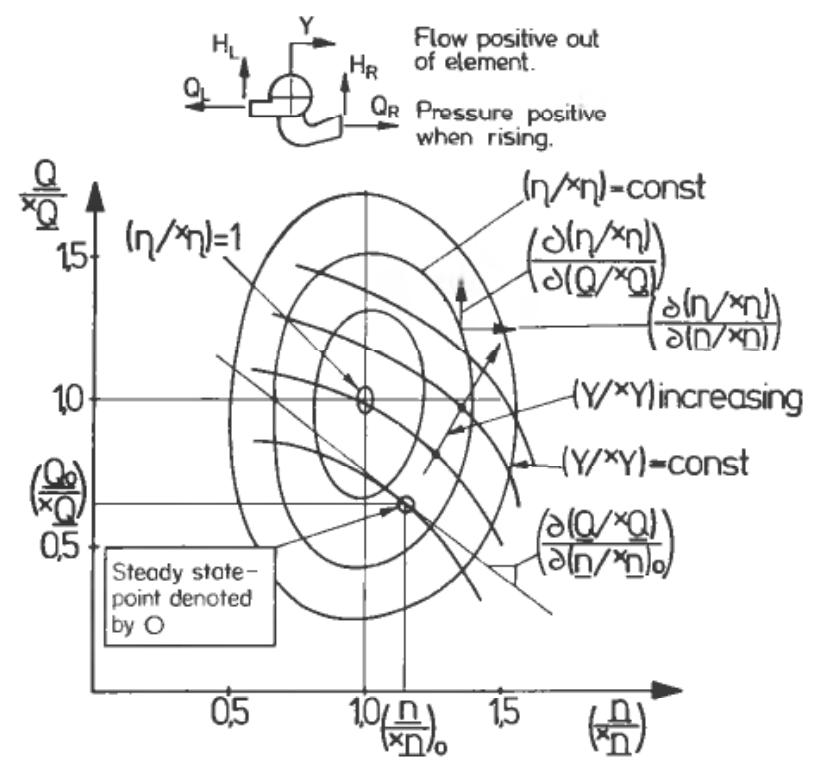

Figure 4. Characteristic diagram for a high head Francis turbine.

The values to be determined from the characteristic diagram are:

$$
\begin{gathered}
Q_{n}=\left({ }^{*} \boldsymbol{Q} \boldsymbol{n}_{0} /\left(\boldsymbol{Q}_{0}{ }^{*} \boldsymbol{n}\right)\right)\left(\frac{\partial\left(\boldsymbol{Q} /{ }^{*} \boldsymbol{Q}\right)}{\partial\left(\boldsymbol{n} /{ }^{*} \boldsymbol{n}\right)}\right)_{0} \\
Q_{y}=\left({ }^{*} \boldsymbol{Q} y_{0} /\left(\boldsymbol{Q}_{0}{ }^{*} y\right)\right)\left(\frac{\partial\left(\boldsymbol{Q} /{ }^{*} \boldsymbol{Q}\right)}{\partial\left(y /{ }^{*} y\right)}\right)_{0} \\
Q_{\phi}=\left({ }^{*} \boldsymbol{Q} \phi_{0} /\left(\boldsymbol{Q}_{0}{ }^{*} \phi\right)\right)\left(\frac{\partial\left(\boldsymbol{Q} /{ }^{*} \boldsymbol{Q}\right)}{\partial\left(\phi /{ }^{*} \phi\right)}\right)_{0}
\end{gathered}
$$

Further the equation for the turbine efficiency will be:

$$
\eta=E_{q} q-\frac{1}{2}\left(E_{q}+E_{n}\right) h+E_{n} n+E_{\phi} \phi
$$

where

$$
\begin{aligned}
& E_{q}=\left({ }^{*} \eta Q_{0} /\left(\eta_{0}{ }^{*} Q\right)\right)\left(\frac{\partial\left(\eta /{ }^{*} \eta\right)}{\partial\left(\boldsymbol{Q} /{ }^{*} Q\right)}\right)_{0} \\
& E_{n}=\left({ }^{*} \eta n_{0} /\left(\eta_{0}{ }^{*} n\right)\right)\left(\frac{\partial\left(\eta /{ }^{*} \eta\right)}{\partial\left(\boldsymbol{n} /{ }^{*} \boldsymbol{n}\right)}\right)_{0} \\
& E_{\phi}=\left({ }^{*} \eta \Phi_{0} /\left(\eta_{0}{ }^{*} \Phi\right)\right)\left(\frac{\partial\left(\eta /{ }^{*} \eta\right)}{\partial\left(\Phi /{ }^{*} \Phi\right)}\right)_{0}
\end{aligned}
$$

The equation for the turbine speed as a function of the generator load including self regulating constant in the grid system may be established as follows.

$$
p /\left(T_{a} s+F_{g}\right)=n .
$$


Further, when denoting the transfer function for the turbine governor by $G$ (PID governor) and the external alternative water level governor by (PID) $H_{0}$ (referring to $1 \mathrm{~m}$ water level), the following general matrix for the turbine/generator element may be established as shown in eqn. (25).

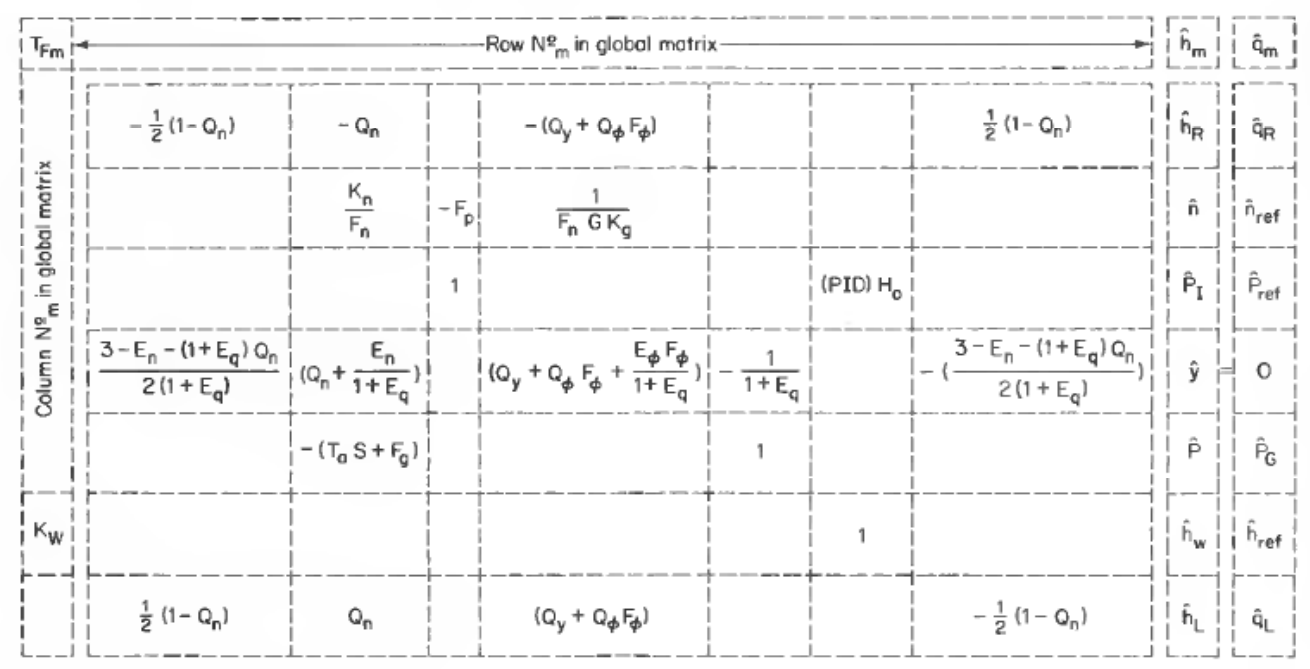

(25)

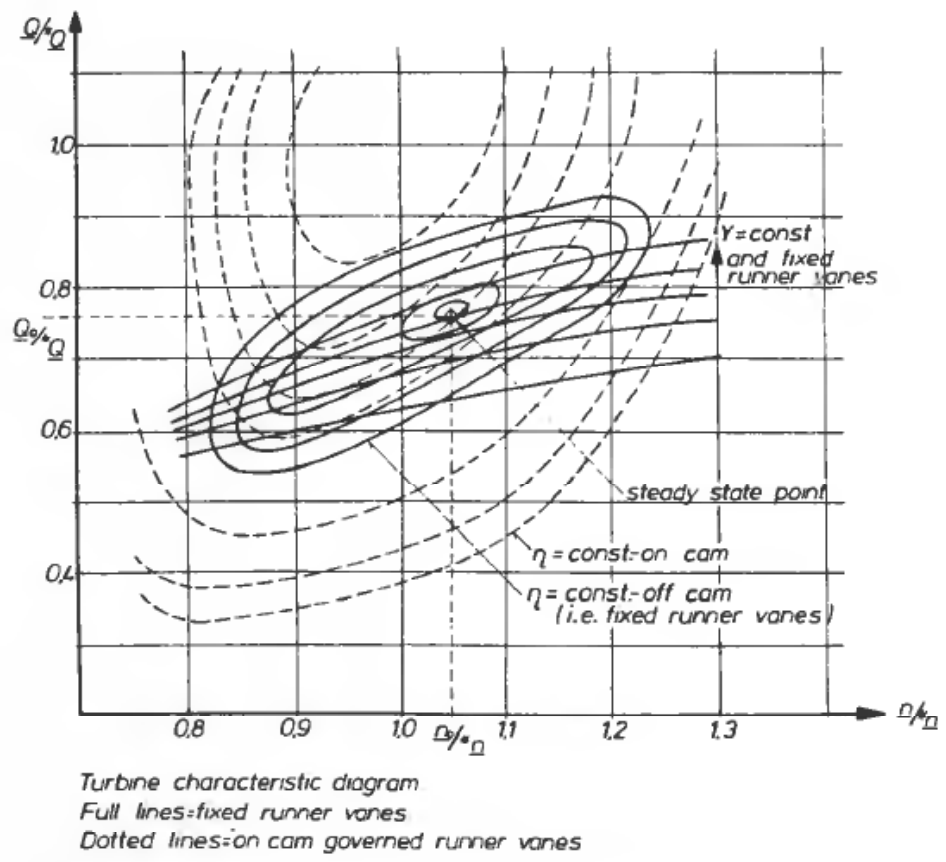

Figure 5. (a) Characteristic diagram for a Kaplan turbine with fixed runner blades ) and on cam with runner blade regulating (------). 
THE DETERMINATION OF THE CONSTANTS BY PARTIAL LINEARIZING OF THE TURBINE CHARACTERISTICS FOR A KAPLAN TURBINE OR KAPLAN BULB - TURBINE
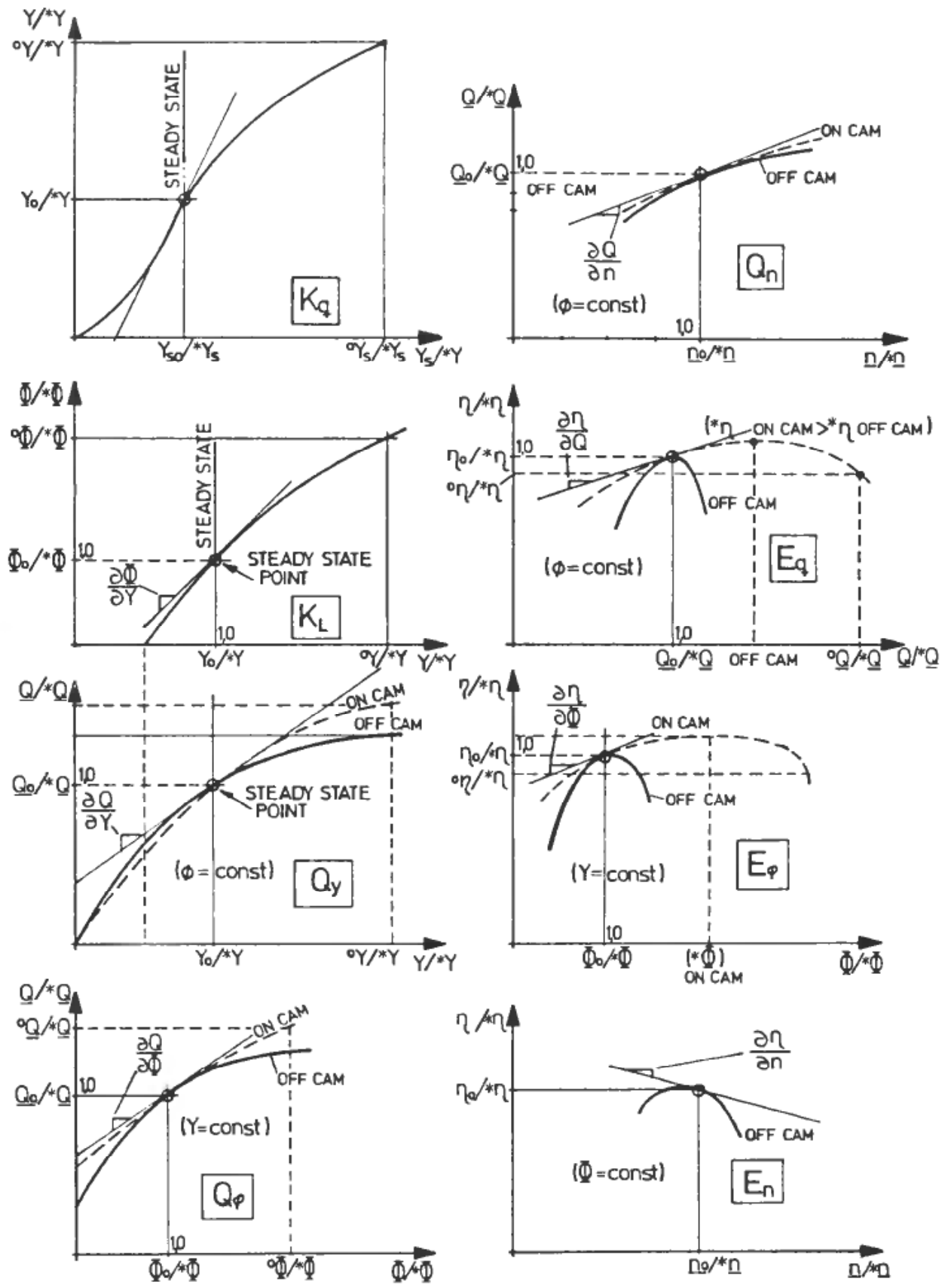

Figure 5. (b) Partial differentiated values to be used in equations (22) and (23) developed by means of the diagram in $(a)$. 


\section{Background for the presented measurements for verification of the} theoretical analysis

In the work presented in Brekke (1984) frequency response measurements have been carried out in six different hydroelectric power plants. Five of the plants were typical high head plants with long tunnel systems while one of the plants was a Kaplan Bulb turbine plant where the output response was measured with relatively good agreement between the theoretical analysis and the measurements. Special interest was paid to the influence of the turbine characteristics and the damping of the surging in the open draught tube gate shaft for this plant.

For the five tunnel plants the pressure response was measured, except for the measurements at Driva Power Plant, where both the output response and the pressure response were measured. In the dissertation (Brekke 1984) all measuring results and theoretical analysis have been presented and in this condensed article only one of the interesting results of the pressure response will be presented. For the pressure response a simplified version of matrix eqn. (25) has been used. This matrix yields:

$$
\left[\begin{array}{ccc}
-\frac{1}{2}\left(1-Q_{n}\right) & -\left(Q_{y}+Q_{\phi} F_{\phi}\right) & \frac{1}{2}\left(1-Q_{n}\right) \\
1 / k_{q} & \\
\frac{1}{2}\left(1-Q_{n}\right) & \left(Q_{y}+Q_{\phi} F_{\phi}\right) & -\frac{1}{2}\left(1-Q_{n}\right)
\end{array}\right] \cdot\left[\begin{array}{c}
h_{L} \\
y \\
h_{R}
\end{array}\right]=\left[\begin{array}{c}
q_{L} \\
y_{\text {ref }} \\
q_{R}
\end{array}\right]
$$

Among the measurements, the result from $\mathbf{J} \phi$ rundland should be regarded as being worthy of special interest.

\subsection{J $\phi$ rundland Power Plant}

The layout of J $\phi$ rundland Power Plant is shown in Fig. 6.

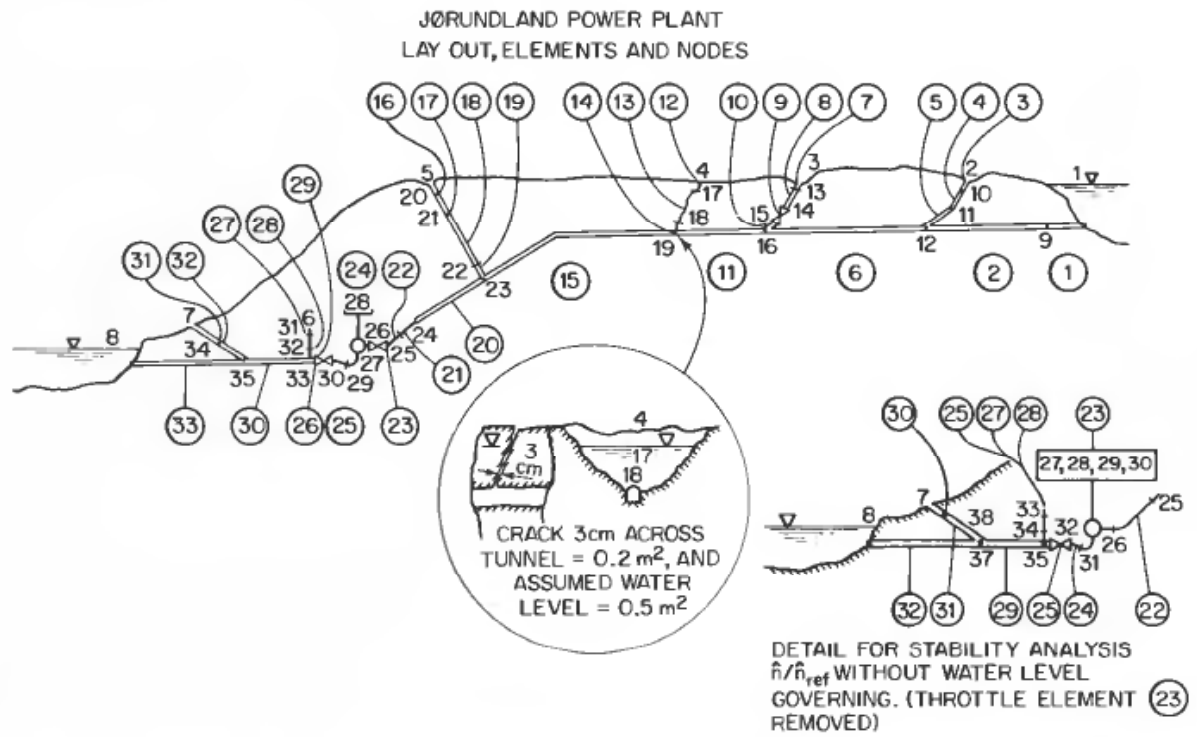

Figure 6. Circled figures denote elements described by the presented submatrices. The other figures denote connecting points or nodes in the global matrix where the submatrices are connected. (Note element No. 24 represents simplified turbine (matrix eqn. 26) with 3 nodes while element 23 in the detail (bottom right) used for the stability analysis represents the matrix eqn. 25 without row and column No. 6, i.e. 6 nodes.) 
The Power Plant is furnished with one Francis turbine with following data.

Nominal output: $\quad{ }^{0} P=55 \mathrm{MW}, \quad{ }^{0} \mathrm{H}=265 \mathrm{~m}, \quad{ }^{0} n=500 \mathrm{RPM}$, ${ }^{0} Q=23 \mathrm{~m}^{3} / \mathrm{sec}$ and ${ }^{0} \eta=0.925$

Best efficiency point: ${ }^{*} P=41 \mathrm{MW},{ }^{*} H=265 \mathrm{~m},{ }^{*} n=500 \mathrm{RPM}$, ${ }^{*} Q=16.8 \mathrm{~m}^{3} / \mathrm{sec}$ and ${ }^{*} \eta=0.94$

Test condition: $\quad P_{0}=38.5 \mathrm{MW}, H_{0}=274 \mathrm{~m}, n_{0}=500 \mathrm{RPM}$, $Q_{0}=15.3 \mathrm{~m}^{3} / \mathrm{sec}$ and $\eta_{0}=0.937$

Of special interest in this plant was the influence from a crack of $0.2 \mathrm{~m}^{2}$ in the rock, which damped down the reflections from the surge shaft elements 12,13 and 14 in Fig. 6. The total length of the tunnel system was about $13 \mathrm{~km}$ with a cross section in the main tunnel of $24 \cdot 2 \mathrm{~m}^{2}$ (theoretical cross section $+10 \%$ ).

In Fig. 7 are shown the measurements with the crack excluded in the theoretical analyses which is shown with full lines in the figure.

In Fig. 8 is shown the analysis with the $0.2 \mathrm{~m}^{2}, 3 \mathrm{~cm}^{2}$ wide crack across the tunnel, which is included in the analysis at a distance of $1632 \mathrm{~m}$ downstream of shaft elements 12, 13 and 14. The crack was detected during blasting because water streamed down into the tunnel and forced work to be stopped for some days. The crack had been left open with no grouting and was filled up with water when the tunnel was filled. The water level of the crack was assumed to be $0.5 \mathrm{~m}^{2}$, but it should be noted that changes of the water level area in the theoretical analysis only made negligible changes to the result

It should also be mentioned that the stability analyses of the governing system showed an improved stability with the crack in the system. With the crack eliminated the stability was not good, whilst excellent stability was obtained with the existing crack. A stability analysis is shown in Fig. 9 and Fig. 10.

\section{General conclusion of the work presented}

(1) The influence of the turbine characteristics introduced in the theoretical analysis showed good agreement with measurements and should not be excluded in the analyses.

(2) The frictional damping of oscillatory flow in tunnels used in the presented theory showed good agreement for blasted tunnels with cross sections varying from $4 \mathrm{~m}^{2}$ to $25 \mathrm{~m}^{2}$ and roughness varying from full profile drilled tunnel walls to rough blasted rock.

(3) The damping function used for joints between tunnels and shafts showed rather good agreement with the presented measurements and also with measurements of other plants.

(4) Measurements of hydroelectric power plants with air accumulator systems showed excellent governing stability compared with similar plants with long surge shafts. (Brekke 1984 and Brekke 1974).

Generally the agreement between theory and measurements in full scale hydroelectric power plants was encourageing for further works in the future in this field.

(Measurements carried out in Spring 1985 in a high head Pelton Power Plant, Aurland I, with a long steel lined penstock proved that the formula used for 


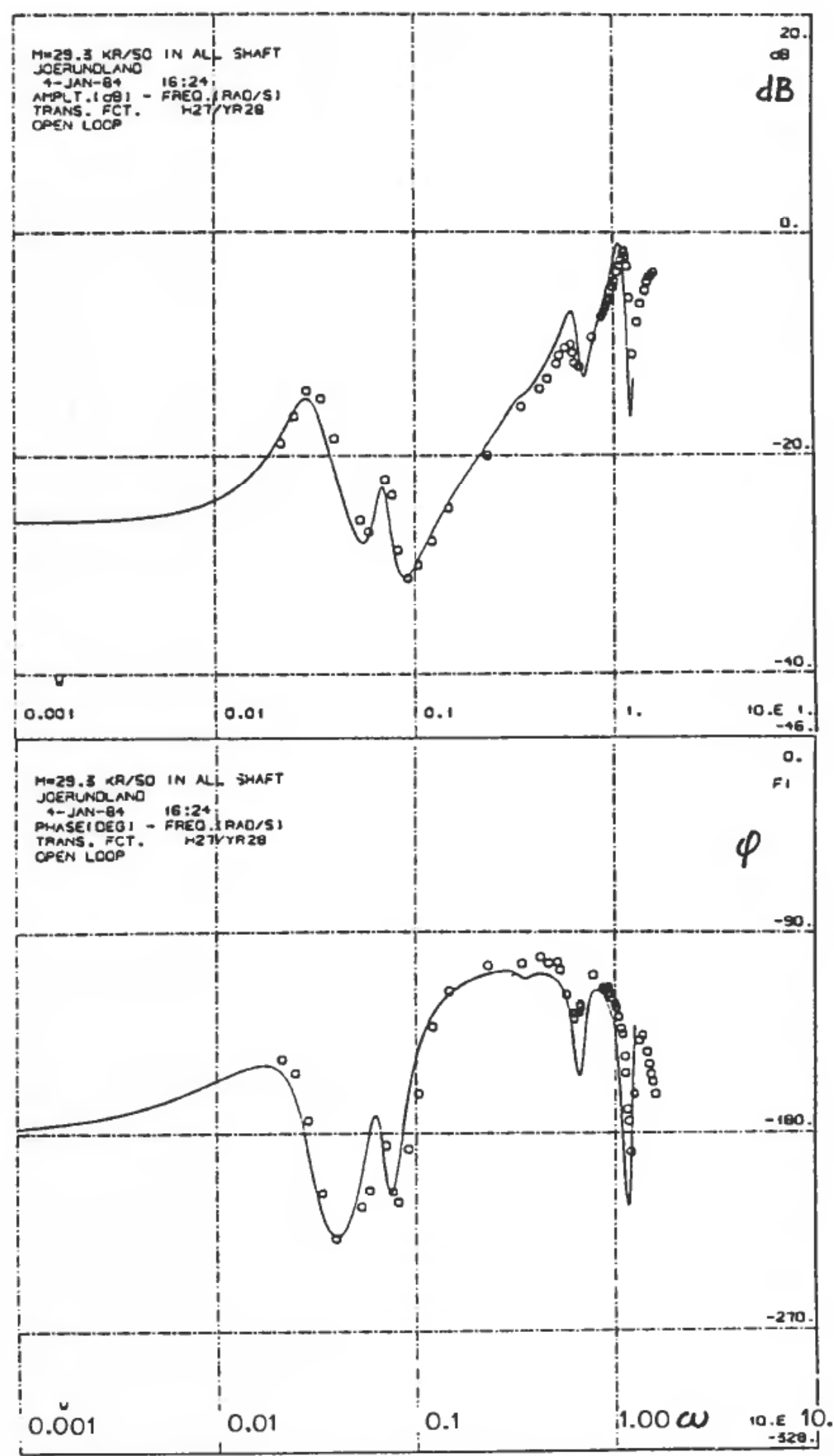

Figure 7. Measurements with the crack included in the theoretical analyses. 
Frequency response analysis of hydroelectric power plants

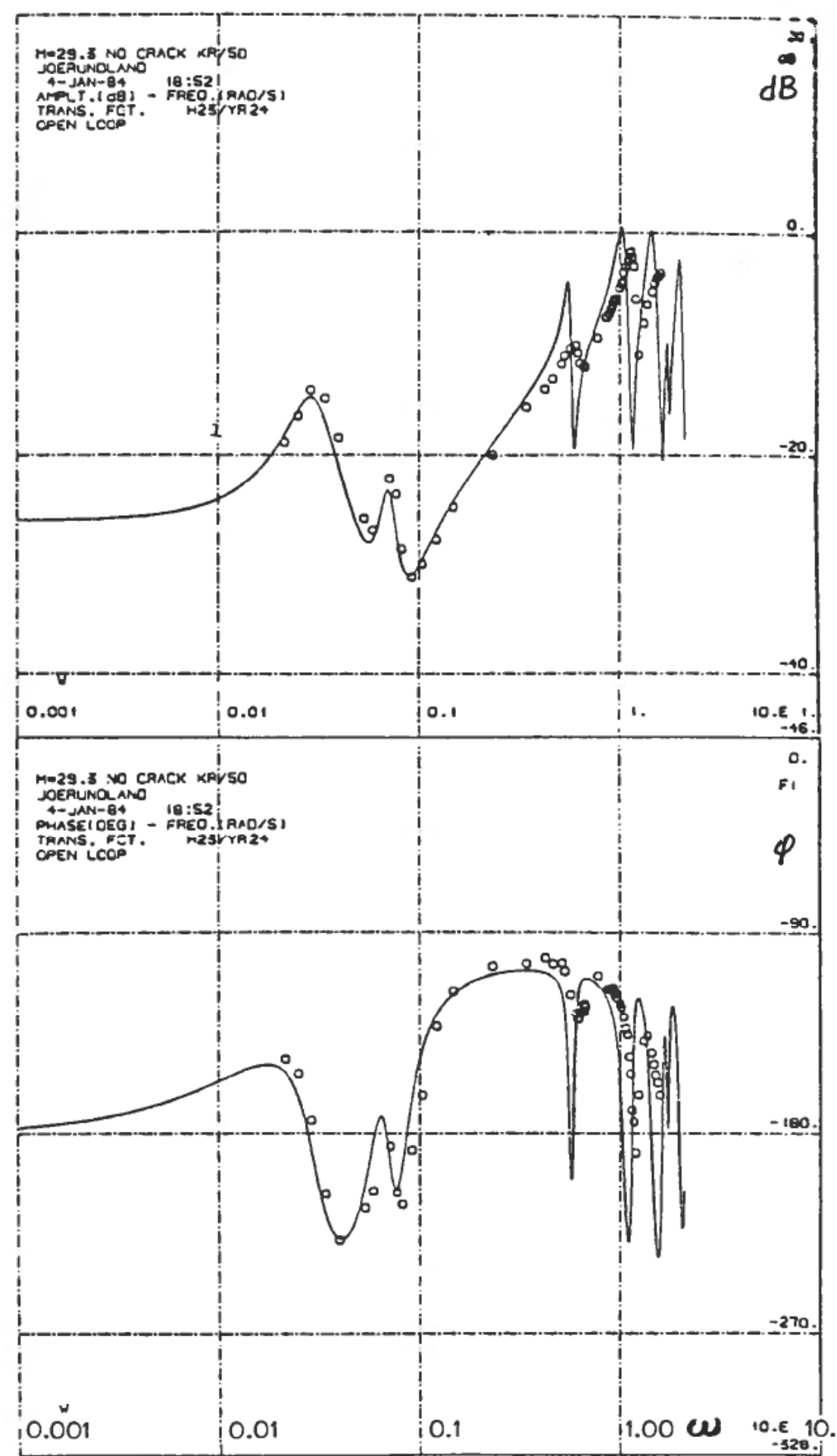

Figure 8. Measurements with the crack excluded in the theoretical analyses. 


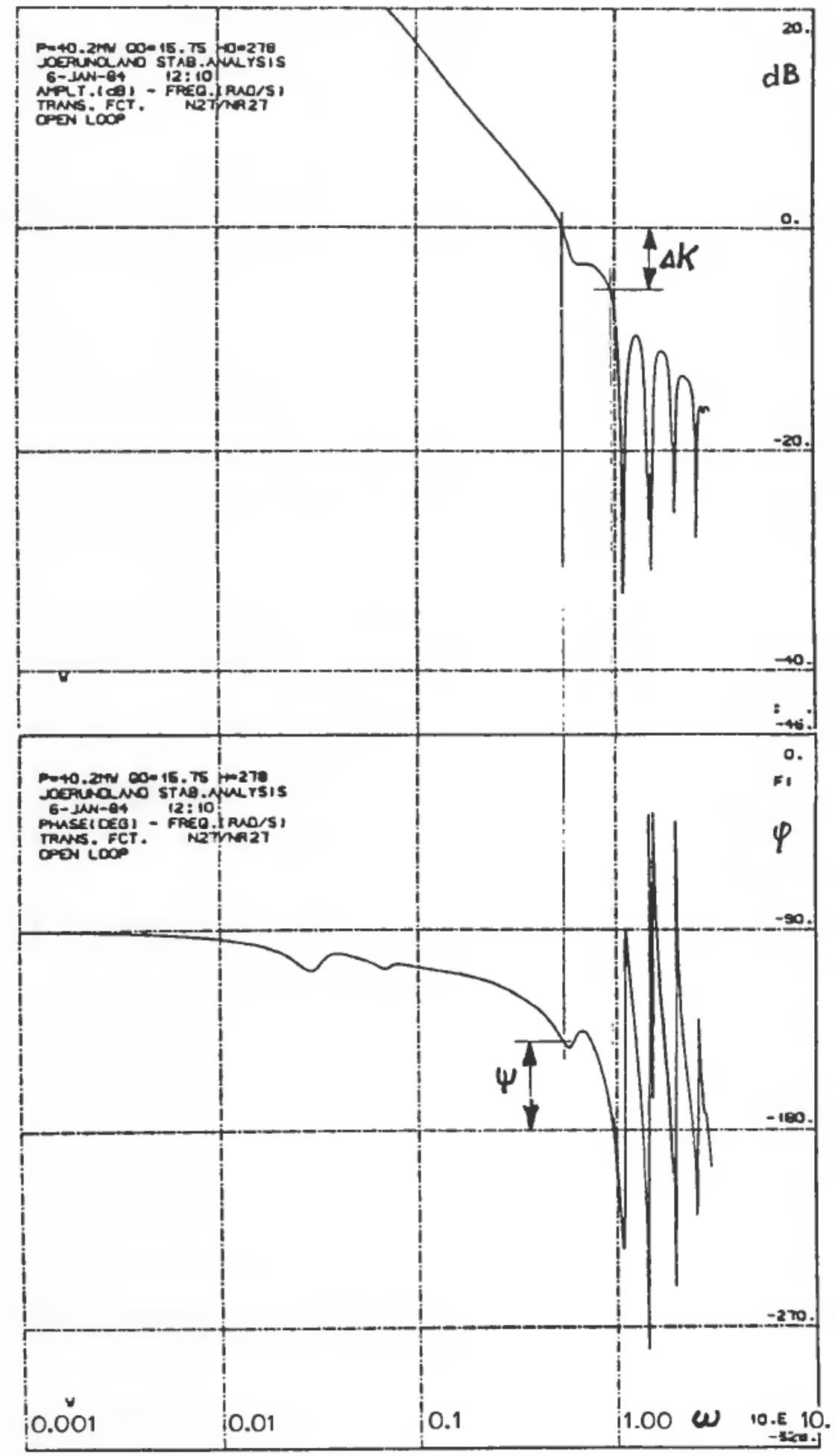

Figure 9. Stability analysis of governing system, with crack. 


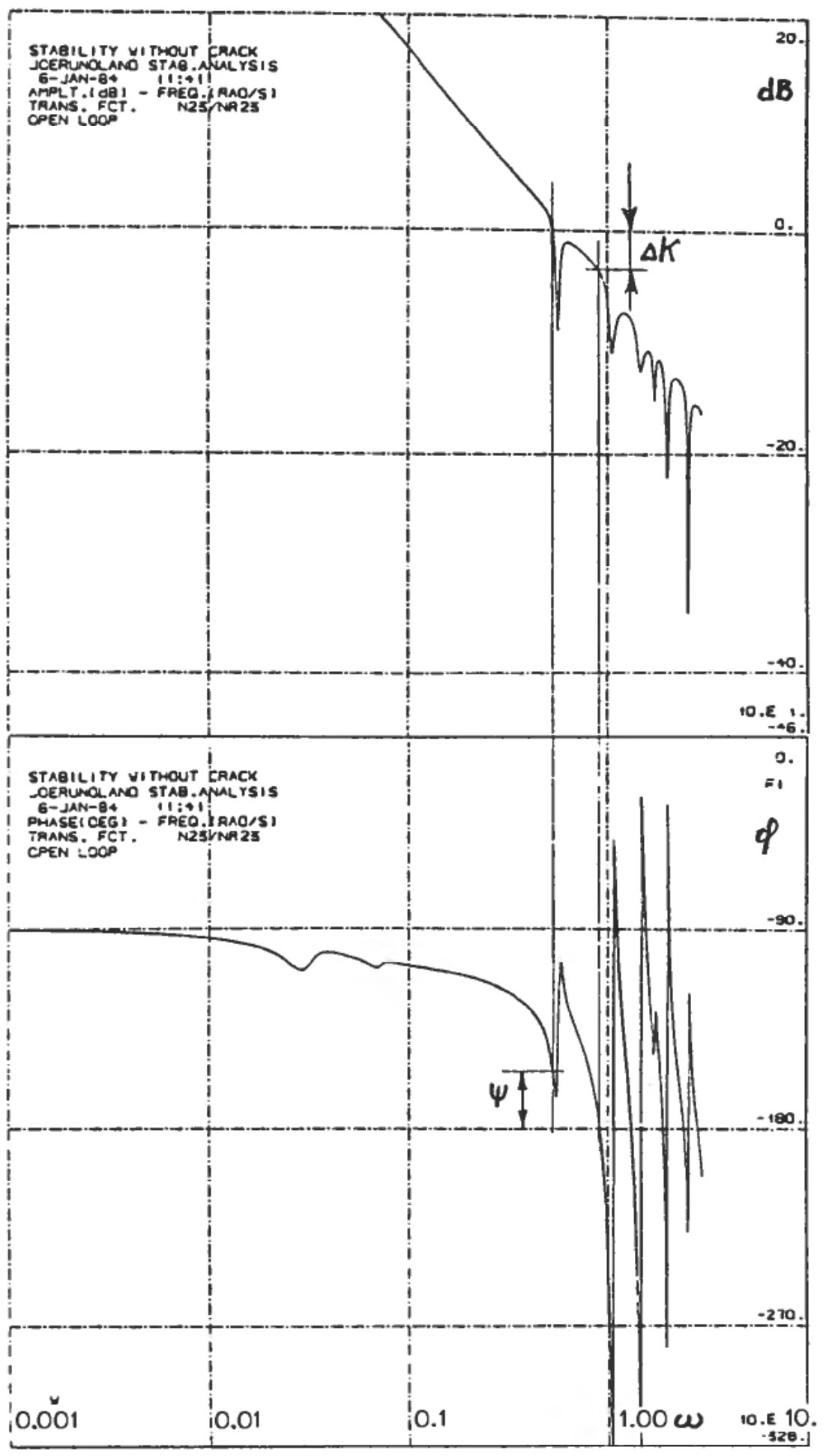

Figure 10. Stability analysis of governing system, without crack. 
damping of oscillatory flow in blasted and full profile drilled tunnels (eqns. 10, 12 and 14) gave good agreement with measurement also for smooth steel lined penstocks.)

APPENDIX

Symbols

$A$ Cross section area

a Wave propagation speed

$\left(\mathrm{m}^{2}\right)$

$(\mathrm{m} / \mathrm{sec})$

(For oscillatory flow $=$ amplitude)

$L \quad$ Length of pipe or tunnel

$h$ Relative pressure $h=H / H_{0}$

$q$ Relative flow $q=Q / Q_{0}$

$h_{f}$ Relative head loss per $\mathrm{m}$ length

$H_{0}$ Mean flow in turbine for reference

$Q_{0}$ Mean flow in turbine for reference

$k$ Friction constant/parameter

$Q_{t}$ Flow in the regarded tunnel or pipe

$h_{w}$ Dimensionless pipe constant (Allievi's constant)

$T_{w}$ Inertia time constant for pipe flow

$\tau$ The wall friction shear stress

$v \quad$ Kinematic viscosity

$\sigma \quad$ Density of water

$h_{m}$ Linearized head loss for oscillations around zero mean flow amplitudes $Q_{m}$

$\hat{h}_{m}$ Relative dimensionless pressure in an arbitrary pond as reference to water level governor

$T_{a}$ Accelerating time constant for rotating masses. (mechanical inertia time)

$F_{g}$ Grid system function

$\Phi \quad$ Runner blade opening

$\hat{y} \quad$ Guide vane of needle opening

$\hat{\phi} \quad d \Phi / \Phi_{0}$ Relative value of runner blade opening

$\hat{y} d Y / Y_{0}$ Relative value of guide vane or needle opening

$o P$ Nominal output of turbine (full load)

$P_{0}$ Steady state output of turbine

$Q_{n}$ Flow/speed constant (linearized value)

$Q_{y}$ Flow/guide vane (needle) stroke constant (linearized value)

$Q_{\phi}$ Flow/runner blade opening constant (linearized value)

$K_{q}$ Ratio constant guide vane/needle-servomotor (linearized)

$K_{L}$ Ratio constant runner blade/guide vane (linearized)

$T_{L}$ Time constant runner blade servomotor

$E_{q}$ Efficiency variation versus flow

$E_{n}$ Efficiency variation versus speed

$E_{\phi}$ Efficiency variation versus runner blade position (m)

$(\mathrm{m} / \mathrm{m})$

$\left(\mathrm{m}^{3} / \mathrm{sec}\right) /\left(\mathrm{m}^{3} / \mathrm{sec}\right)$

$(\mathrm{m} / \mathrm{m})$

(m)

$\left(\mathrm{m}^{3} / \mathrm{sec}\right)$

$\left(\mathrm{m}^{3} / \mathrm{sec}\right)$

(sec)

$\left(\mathrm{N} / \mathrm{m}^{2}\right)$

$\left(\mathrm{m}^{2} / \mathrm{s}\right)$

$\left(\mathrm{kg} / \mathrm{m}^{3}\right)$

$(\mathrm{m} / \mathrm{m})$

$(\mathrm{m} / \mathrm{m})$

(sec)

(MW)

(MW)

(sec) 
$n_{0}$ Speed, steady state

${ }^{0} n$ Speed, nominal

$b_{t}$ Temporary speed drop constant (in governor)

$T_{N}$ Derivative time constant

(sec)

$T_{D}$ Integrating time constant

$T_{0}$ Time constant in additional servomotor (two stage system)

$T_{y}$ Time constant in main servomotor

$T_{p}$ Time constant in electrohydraulic amplifier

$b_{p}$ Permanent speed drop constant

$K_{n} K_{p}, K_{w}$ Constants used in computer for including or excluding feed back signals

\section{REFERENCES}

Brekke, H. (May 1984). A stability study on Hydroelectric Power Plant governing including the influence from a quasi nonlinear damping of oscillatory flow and from the turbine characteristics. Dissertation, The Norwegian Institute of Technology, adr. 7034 Trondheim-NTH.

BREKKE, H. (1974). Induced hydraulic resonance analysis on a Francis turbine power plant with an air cushioned high pressure tunnel system. IAHR Symposium, Vienna, Austria, 1974.

JoNSSON, I. G. (1978). A new approach to oscillatory rough turbulent boundary layers. Series Paper 17 (1978). I.S.V.A., Technical University of Denmark.

KÖNGETER, J. (1980). Rohrreibungsverluste einer oscillierenden turbulente Strömung in einem Kreisrohr konstanten Querschnitts. Mitteilung Nr. 95, Technische Universität Berlin.

KNOTT, G. F., and Flower, J. O. (1980). Measurement of energy losses in oscillatory flow through a pipe exit. Applied Ocean Research, 2, vol. 2, no 4, pp. 155-165 